\title{
The root cause of the attentional blink: First-target processing or disruption of input control?
}

\author{
Hayley E. P. Lagroix • Thomas M. Spalek • Brad Wyble • \\ Ali Jannati • Vincent Di Lollo
}

Published online: 15 August 2012

(C) Psychonomic Society, Inc. 2012

\begin{abstract}
Identification of the second of two targets (T2) is impaired when presented shortly after the first (T1). T1-based theories ascribe this attentional blink $(\mathrm{AB})$ to a T1-initiated period of inattention. Distractor-based theories ascribe it to a disruption of input control caused by post-T1 distractors. The finding that an $\mathrm{AB}$ occurs without intertarget distractors (Nieuwenstein, Potter, \& Theeuwes, Journal of Experimental Psychology: Human Perception and Performance 35:159-169, 2009) seemingly disconfirms distractor-based theories. The present experiments addressed different ways in which distractor-based theories might account for that finding. Intertarget events were varied in four experiments. Experiment 1 replicated Nieuwenstein, Potter, and Theeuwes's findings. The next two experiments tested two ways (lack of visual stimulation, violation of expectation) in which the blank intertarget interval might cause an AB. Experiment 4 explored whether backward-masking of $\mathrm{T} 1$ can account entirely for the larger AB obtained with intervening distractors or whether distractors also disrupt input control. The results disconfirm predictions from distractor-based theories and support the claim of T1-based theories that $\mathrm{T} 1$ processing alone is sufficient for the $\mathrm{AB}$. Simulations based on the eSTST (Wyble, Bowman, \& Nieuwenstein, Journal of Experimental Psychology: Human Perception and Performance 35:787-807, 2009) and the B\&B models (Olivers \& Meeter, Psychological Research, 115, 8368632008 ) were compared. Predictions were more accurate from
\end{abstract}

H. E. P. Lagroix $(\bowtie) \cdot$ T. M. Spalek · A. Jannati $\cdot$ V. Di Lollo Department of Psychology, Simon Fraser University, 8888 University Drive,

Burnaby, BC, Canada V5A 1S6

e-mail: hlagroix@sfu.ca

B. Wyble

Syracuse University,

Syracuse, NY, USA the T1-based theory (eSTST) than from the distractor-based theory $(\mathrm{B} \& \mathrm{~B})$.

Keywords Attentional blink · Selective attention · Visual attention

Identification of the second of two sequential targets (T2) is impaired if it is presented shortly after the first (T1). This T2 deficit, known as the attentional blink (AB; Broadbent \& Broadbent, 1987; Raymond, Shapiro, \& Arnell, 1992), is most pronounced when the temporal separation between the two targets is short (200-300 ms), with performance improving as the separation is increased to about $700 \mathrm{~ms}$ (Raymond et al., 1992). In most studies, the two targets (e.g., letters) are inserted in a stream of distractors (e.g., digits) displayed in rapid serial visual presentation (RSVP; Potter \& Levy, 1969). The temporal separation between the two targets is varied in steps of about $100 \mathrm{~ms}$, with each step denoted as a separate lag. Thus, the term Lag 1 indicates that T2 was presented directly after T1, and the term $\operatorname{Lag} 3$ indicates that two distractors intervened between $\mathrm{T} 1$ and $\mathrm{T} 2$.

\section{Theoretical accounts of the attentional blink}

Many theoretical accounts, both formal and qualitative, have been proposed to explain the processing limitations revealed by the $\mathrm{AB}$. Recent reviews have been provided by Dux and Marois (2009) and Martens and Wyble (2010). Here, we confine our survey to studies that set the stage for the present experiments, which were designed to distinguish between two classes of theory: T1 based and distractor based. According to T1-based theories, T1 processing alone is sufficient to cause the AB. In distractor-based theories, on the other hand, the requirement to process T1 
is not sufficient; the presence of at least one distractor following $\mathrm{T} 1$ is regarded as essential.

\section{T1-based theories}

The class of T1-based theories encompasses a number of individual theories that differ as to the specific cause of the AB. These include resource depletion (e.g., Ward, Duncan, \& Shapiro, 1996), delayed selection (e.g., Nieuwenstein, Potter, \& Theeuwes, 2009), and working memory consolidation (e.g., Chun \& Potter, 1995; Jolicœur \& Dell'Acqua, 1998; Wyble, Bowman, \& Nieuwenstein, 2009). Despite individual variations, these theories share the common tenet that the $\mathrm{AB}$ stems from some process or event directly associated with $\mathrm{T} 1$ processing.

The prototypical exemplar of T1-based theories is the two-stage model proposed by Chun and Potter (1995). Incoming stimuli are said to be processed in parallel in a high-capacity first stage where potential targets are rapidly identified. At this stage, identity information is volatile and vulnerable to both decay and overwriting by trailing items. Targets tentatively identified in stage 1 are consolidated in a second stage where processing is said to be serial and capacity limited, in that only a single target can be processed at a time. The $\mathrm{AB}$ occurs if $\mathrm{T} 2$ arrives while stage 2 is still busy processing $\mathrm{T} 1$. In that case, $\mathrm{T} 2$ is delayed in stage 1 , where it is vulnerable to decay and overwriting by subsequent items.

Some elements of Chun and Potter's (1995) two-stage model have been incorporated into computational accounts of the AB. For example, the episodic simultaneous type, serial token (eSTST) model of Wyble et al. (2009; see also Bowman \& Wyble, 2007) makes use of the two-stage architecture and transient attentional mechanism that were part of the Chun and Potter model.

However, the eSTST model provides a novel theoretical rationale for the two-stage architecture by proposing that the $\mathrm{AB}$ reflects processes involved in creating episodically distinct representations within working memory. In the first stage (the input layer), abstract identity information about each stimulus (i.e., its type; Kanwisher, 1987; Mozer, 1989) is extracted. For a stimulus to be reported correctly, however, it must gain access to a later encoding stage where its identity information is bound to a token (Kanwisher, 1987; Mozer, 1989) and is thereby encoded into working memory.

For a type representation to be bound to a token, it must be enhanced by a transient attentional mechanism that amplifies the strength of the type signal. This mechanism is triggered upon detection of a target, and its activity lasts for approximately $200 \mathrm{~ms}$, provided that no additional targets are presented during this excitatory phase. An inhibitory phase then follows, during which attention is suppressed while the target undergoes the binding process. This T1- triggered suppression of attention extends over several hundred milliseconds and mediates the $\mathrm{AB}$ by preventing the $\mathrm{T} 2$ type from being bound to a token until $\mathrm{T} 1$ tokenization is complete. As with other T1-based theories, this delay in T2 processing leaves it vulnerable to decay and interference by trailing items.

In summary, $\mathrm{T} 1$-based theories see the $\mathrm{AB}$ as arising from $\mathrm{T} 1$ processing. The only role assigned to distractors presented between $\mathrm{T} 1$ and $\mathrm{T} 2$ (hereafter referred to as intervening distractors) is as masks for $\mathrm{T} 1$. Such masking may increase the difficulty of $\mathrm{T} 1$ processing, thereby increasing the length and magnitude of the AB.

\section{Distractor-based theories}

The class of distractor-based theories encompasses several models, each with somewhat different accounts of the $A B$ (e.g., Di Lollo, Kawahara, Ghorashi, \& Enns, 2005; Olivers, van der Stigchel, \& Hulleman, 2007; Olivers \& Meeter, 2008; Raymond et al., 1992). What all these accounts have in common is the claim that the period of inattention that is the signature of the $\mathrm{AB}$ is triggered not by the requirement to process $\mathrm{T} 1$ but by the disruptive effect of distractors intervening between the two targets.

The first distractor-based theory was proposed by Raymond et al. (1992). Detection of a target (e.g., T1) is said to initiate an attentional episode by opening a gate to a sensory store from which items can be selected for identification. While the gate is open, any ensuing items also gain access to the sensory store. The presence in the sensory store of features from both the target and trailing distractors may cause confusion, thus interfering with target selection. When this happens, the attentional gate is locked, and a suppressive mechanism is activated to prevent further items from entering the sensory store until processing of $\mathrm{T} 1$ has been completed. If $\mathrm{T} 2$ arrives after a distractor has caused the gate to be locked, an $\mathrm{AB}$ occurs because the processing of $\mathrm{T} 2$ is suppressed. This model is clearly distractor based, in that if $\mathrm{T} 1$ is not followed by at least one distractor, the gate is not locked, and the $\mathrm{AB}$ does not occur.

This distractor-based account was not subject to serious inquiry until it was demonstrated that no $\mathrm{AB}$ is observed when multiple targets (e.g., T1, T2, T3) appear in direct succession (Di Lollo et al., 2005; Kawahara, Kumada, \& Di Lollo, 2006; Nieuwenstein \& Potter, 2006; Olivers et al., 2007; Olivers \& Meeter, 2008; Potter, Nieuwenstein, \& Strohminger, 2008). The absence of an AB in these studies stands in stark contrast to the finding in conventional studies in which a substantial $A B$ is observed when as few as two targets are separated by a single distractor (T1, D, T2). This finding ignited a new debate about the underlying cause of the $\mathrm{AB}$ and promoted the development of several new models, some of which were also computational in nature. 
The temporary loss of control (TLC) model (Di Lollo et al., 2005) proposes that target selection is governed by an input filter configured endogenously to pass targets and reject distractors (see also Ghorashi, Zuvic, Visser, \& Di Lollo, 2003; Kawahara et al., 2006; Visser, Bischof, \& Di Lollo, 2004). A central processor is charged with both actively maintaining the input filter (by issuing repeated signals to the filter) and encoding targets into working memory. Critically, the central processor can execute only one of these operations at a time. When a target is detected, the central processor switches from issuing maintenance signals to coordinating the target's processing. In the absence of maintenance signals, the filter becomes vulnerable to exogenous disruption by stimuli (e.g., distractors) that do not match the filter's current configuration. If the item following $\mathrm{T} 1$ is another target, the filter will not be reconfigured, because targets fit with the existing filter configuration. Targets will thus gain direct access to working memory, thereby avoiding the $\mathrm{AB}$. If, however, the item following $\mathrm{T} 1$ is a distractor, the filter is disrupted. Any targets appearing after this disruption, while $\mathrm{T} 1$ is still being encoded, will not match the filter's configuration and, therefore, will be delayed until T1 processing is complete. It is only then that the input filter can be reconfigured by the central processor to once again pass targets. During this delay, T2 is vulnerable to both decay and masking, which causes the AB.

A computational model that is also distractor based is the boost and bounce (B\&B) model of Olivers et al. (2007; Olivers \& Meeter, 2008), which has a two-stage architecture. In this account, all items in the RSVP stream are said to undergo sensory processing during which their perceptual features and semantic and categorical information are activated. Only a small subset of these items, however, can gain access to the second, working memory encoding stage. For an item to be encoded into working memory where it is available for report, it must be attentionally enhanced, or "boosted," by a gating mechanism. Stimuli that match the attentional set established by task instructions are boosted, whereas stimuli that do not (i.e., distractors) are inhibited, or "bounced." Detection of T1 initiates a boost of attention that vaults it into working memory. Because the boosting mechanism is sluggish, the item that immediately follows $\mathrm{T} 1$ is also boosted, regardless of whether it is a target or a distractor. If that item is another target (i.e., T2), it triggers another boost and is encoded into working memory along with T1. On the other hand, if that item is a distractor, a strong but transient suppression (bounce) is initiated that inhibits the processing of the next few items to prevent further distractors from being encoded into working memory. If one of those items happens to be $\mathrm{T} 2$, an $\mathrm{AB}$ results, since the distractor-induced inhibition prevents $\mathrm{T} 2$ from receiving the boost needed for working memory encoding.
Another computationally formalized distractor-based account of the $\mathrm{AB}$ has been proposed by Taatgen, Juvina, Schipper, Borst, and Martens (2009). In the threaded cognition model, which is built using the ACT-R framework (Anderson \& Lebiere, 1998), the detection of a distractor during ongoing processing of $\mathrm{T} 1$ elicits a protective attentional mechanism that blocks processing of new stimuli until $\mathrm{T} 1$ processing has been completed. This account shares some similarities with $\mathrm{T} 1$-based theories in that the duration of the $\mathrm{AB}$ is tied directly to $\mathrm{T} 1$ processing. However, in the threaded cognition model, the $\mathrm{AB}$ is elicited specifically by the presence of a post-T1 distractor; hence, it should be classified as a distractor-based model.

\section{The root cause of the AB: $\mathrm{T} 1$ processing or distractor interference?}

T1-based and distractor-based theories have been the subject of considerable debate. Studies that attempted to distinguish between the two approaches have typically done so in one of two ways: (1) by manipulating T1 difficulty and/or T1 processing demands or (2) by manipulating the events that intervene between the two targets.

\section{Manipulations of T1difficulty}

Numerous studies have examined the magnitude of the AB in relation to T1-processing difficulty. The outcomes of these studies have been mixed. Some studies found the $\mathrm{AB}$ to vary with $\mathrm{T} 1$ difficulty (Akyürek, Hommel, \& Jolicœur, 2007; Colzato, Spape, Pannebakker, \& Hommel, 2007; Dux \& Coltheart, 2005; Grandison, Ghirardelli, \& Egeth, 1997; Jolicœur, 1998, 1999; McAuliffe \& Knowlton, 2000; Ouimet \& Jolicœur, 2007; Seiffert \& Di Lollo, 1997; Visser, 2007). Other studies found no such relationship (Akyürek \& Hommel, 2005, 2006; McLaughlin, Shore, \& Klein, 2001; Shapiro, Raymond, \& Arnell, 1994; Ward, Duncan, \&Shapiro, 1997). Although several hypotheses have been advanced to account for this discrepancy, there is no general agreement as to how, or even whether, $\mathrm{T} 1$ processing demands modulate the $\mathrm{AB}$ (McLaughlin et al., 2001; Olivers \& Meeter, 2008; Olson, Chun, \& Anderson, 2001; Visser, 2007).

Even if the reason for these mixed results could be determined, it could still be argued that manipulating $\mathrm{T} 1$ processing as a means of distinguishing between T1-based and distractor-based theories may not be an ideal strategy. First, evidence that T1-difficulty modulates the $\mathrm{AB}$, while consistent with T1-based models, is not necessarily inconsistent with distractor-based models. In fact, distractor-based models predict that $\mathrm{T} 1$ processing plays an essential role in the $\mathrm{AB}$ : It is only during $\mathrm{T} 1$ processing that distractors can 
disrupt attention and cause an AB. It could be argued, therefore, that some distractor-based theories would predict modulations of the $\mathrm{AB}$ with manipulations of $\mathrm{T} 1$ difficulty. For example, the TLC model would predict that input control would be lost for a longer period of time when T1 difficulty is higher. This is because input control cannot be reestablished until $\mathrm{T} 1$ processing is complete.

Clearly, given that $\mathrm{T} 1$ difficulty plays a role in both classes of theory, it cannot be used as a discriminative criterion. What is needed is a factor that is hypothesized to affect the $A B$ in one class of theory but not in the other. The presence/absence of distractors intervening between $\mathrm{T} 1$ and $\mathrm{T} 2$ is such a factor. $\mathrm{T} 1$-based theories predict that an $\mathrm{AB}$ should occur, regardless of the presence or absence of intervening distractors. The sole requirement is that $\mathrm{T} 1$ be processed. In contrast, distractorbased theories predict that an $\mathrm{AB}$ should be in evidence only when distractors intervene between the two targets.

Manipulations of events intervening between the targets

Several studies have examined the $\mathrm{AB}$ under conditions in which the intertarget distractors were replaced with blank intervals of corresponding duration. These studies have found that an $\mathrm{AB}$ occurs in the absence of intervening distractors (Nieuwenstein , Potter, \& Theeuwes, 2009; Nieuwenstein, Van der Burg, Theeuws, Wyble, \& Potter, 2009; Visser, 2007; Wyble, Potter, Bowman, \& Nieuwenstein, 2011). A temporal gap was inserted between the two targets in several other studies, (e.g., Chun \& Potter, 1995; Raymond et al., 1992; Seiffert \& Di Lollo, 1997). However, the fact that distractors were also presented during the intertarget interval precludes any conclusion as to whether an $\mathrm{AB}$ can be obtained without intervening distractors.

The outcomes of these studies seem to indicate that the presence of distractors during the intertarget interval is not essential for the $\mathrm{AB}$. On the face of it, this finding runs counter to the central assumption of distractor-based models. However, this need not be regarded as decisive evidence against those models. The possibility remains that the intertarget gap may be distracting in and of itself. Namely, it is possible that there is something about the gap, besides the mere absence of distractors, which played a role in causing the observed $\mathrm{AB}$. For example, the unexpected gap may itself be a source of distraction, in some way similar to a distractor item. The present work explores this, as well as other possible sources of distraction.

\section{Objectives of the present work}

The principal objective of the present work was to examine differential predictions from the two classes of theory regarding the effect of intertarget stimuli on the occurrence of the AB. In a nutshell, while differing in detail, all distractor-based theories share the claim that $\mathrm{T} 1$ processing alone is not sufficient for producing the $\mathrm{AB}$. Instead, they all claim that it is the presence of intervening distractors that is essential. In contrast, T1-based theories assign no role to distractors in causing the $\mathrm{AB}$ : $\mathrm{T} 1$ processing alone is sufficient. In addition to these qualitative predictions, we have selected one exemplar from each class of theory that makes formal predictions regarding the role of intertarget events: the distractor-based B\&B model (Olivers \& Meeter, 2008) and the T1-based eSTST model of Wyble et al. (2009). Computer simulations based on these models are illustrated along with the empirical data for each experiment. It should be emphasized that neither of these models is being fit to the data. Instead, the parameters are exactly the same as in the original published studies. Thus, all of the simulations presented here are de novo predictions in that these experimental data did not exist at the time the models were published.

Experiments 1-3 addressed possible reasons why an $\mathrm{AB}$ may occur when the distractors intervening between the two targets are replaced by blanks. Experiment 2 explored the option that the lack of visual stimulation during the intertarget interval may have caused attention to wander, either in external space or inward to other mental processes, causing attention to be unavailable upon the presentation of T2. Experiment 3 explored the option that the blank interval may have come as a surprise, thereby disrupting attention.

These options were explored with a method that differed from that used in earlier studies in which the intervening distractors were omitted from the RSVP stream. The dependent measure used in the earlier studies was the accuracy of T2 identification. That measure, however, is subject to ceiling constraints imposed by the $100 \%$ limit of the response scale. The effect of such a response ceiling on the experimental outcome can be significant (e.g., Ghorashi, Enns, Spalek, \& Di Lollo, 2009; Jannati, Spalek, \& Di Lollo, 2011; Jannati, Spalek, Lagroix, \& Di Lollo, 2012).

Ceiling constraints can be avoided by using a dynamic threshold-tracking procedure known as parameter estimation by sequential testing (PEST; Taylor \& Creelman, 1967). In the present work, the PEST procedure was used to find the critical interstimulus interval $\left(\mathrm{ISI}_{\mathrm{C}}\right.$ ) between $\mathrm{T} 2$ and the trailing mask at which any given participant could identify T2 approximately $80 \%$ of the time.

Experiment 1 was designed to confirm that the $\mathrm{AB}$ obtained with the PEST procedure is homologous with that obtained using conventional accuracy measures. A second, just as important, objective was to establish a baseline for comparing the results of subsequent experiments.

\section{Experiment 1}

Experiment 1 contained two conditions. In one, the stimuli consisted of a conventional RSVP stream with distractors 
intervening between the two targets. In the other, the intervening distractors were replaced with blank frames of corresponding duration. As was noted above, the principal objective was to verify that the pattern of results obtained in earlier studies with accuracy of T2 identification as the dependent measure (e.g., Nieuwenstein, Potter, \& Theeuwes, 2009) is obtained when the dependent measure is the ISI $_{C}$ obtained with the PEST procedure. This procedure has a crucial advantage in reducing the ceiling effects, which can conceal the presence of an $\mathrm{AB}$.

Method

\section{Participants}

Twenty-eight undergraduate students participated for course credit. All reported normal or corrected-to normal vision and were naïve as to the purpose of the experiment. In this and all subsequent experiments, participants gave written informed consent prior to the experiment. The Office of Research Ethics at Simon Fraser University approved this study. The 28 participants were randomly assigned to two groups of 14 participants each: the intervening-distractors and the intervening-gap groups.

\section{Apparatus and stimuli}

Stimuli were displayed on an NEC AccuSync CRT monitor with a resolution of $600 \times 800$ pixels at a refresh rate of $140 \mathrm{~Hz}$. All stimuli were black (20-point Helvetica font; RGB $0,0,0 ; 0.9 \mathrm{~cd} / \mathrm{m}^{2}$ ) and were presented in the center of the screen against a gray background (RGB 90, 90, 90; $15.0 \mathrm{~cd} / \mathrm{m}^{2}$ ). The RSVP stream contained a variable number of digit distractors (2-9) and two uppercase letter targets (T1 and T2) selected randomly without replacement from the English alphabet, except I, O, W, and M. All stimuli subtended approximately $0.6^{\circ}$ vertically. Following Nieuwenstein, Potter, and Theeuwes (2009), T2 was followed by a pattern mask consisting of a white square in which a circle, a pound sign, and three additional line segments were drawn in black (see Fig. 1a, b). The experiment was programmed in E-Prime (Version 2.0; Psychological Software Tools, Pittsburgh, PA).

\section{Design and procedure}

The experiment was run in a dimly lit room. At the beginning of a session, participants were required to read the instructions displayed on the screen and were invited to ask questions. All
Fig. 1 a and b Schematic representation of the sequence of events in the intervening distractors and intervening gap conditions, respectively, in Experiment 1. Each square represents one 7.14-ms frame, except for the mask, which was presented for $400 \mathrm{~ms}$. The small gap between each square represents a blank ISI of $93 \mathrm{~ms}$. The T2-mask ISI was governed by PEST which converged on the critical ISI $\left(\mathrm{ISI}_{\mathrm{C}}\right)$ required for $80 \%$ correct T2 identifications. $\mathbf{c}$ Mean $\mathrm{ISI}_{\mathrm{C}}$ as a function of lag in Experiment 1. Interv. = intervening; ISI = interstimulus interval; $\mathrm{ms}=$ milliseconds. Error bars indicate standard errors of the means. $\mathbf{d}$ and e Computer simulations from the eSTST and the $\mathrm{B} \& \mathrm{~B}$ models, respectively. See the text for explanation
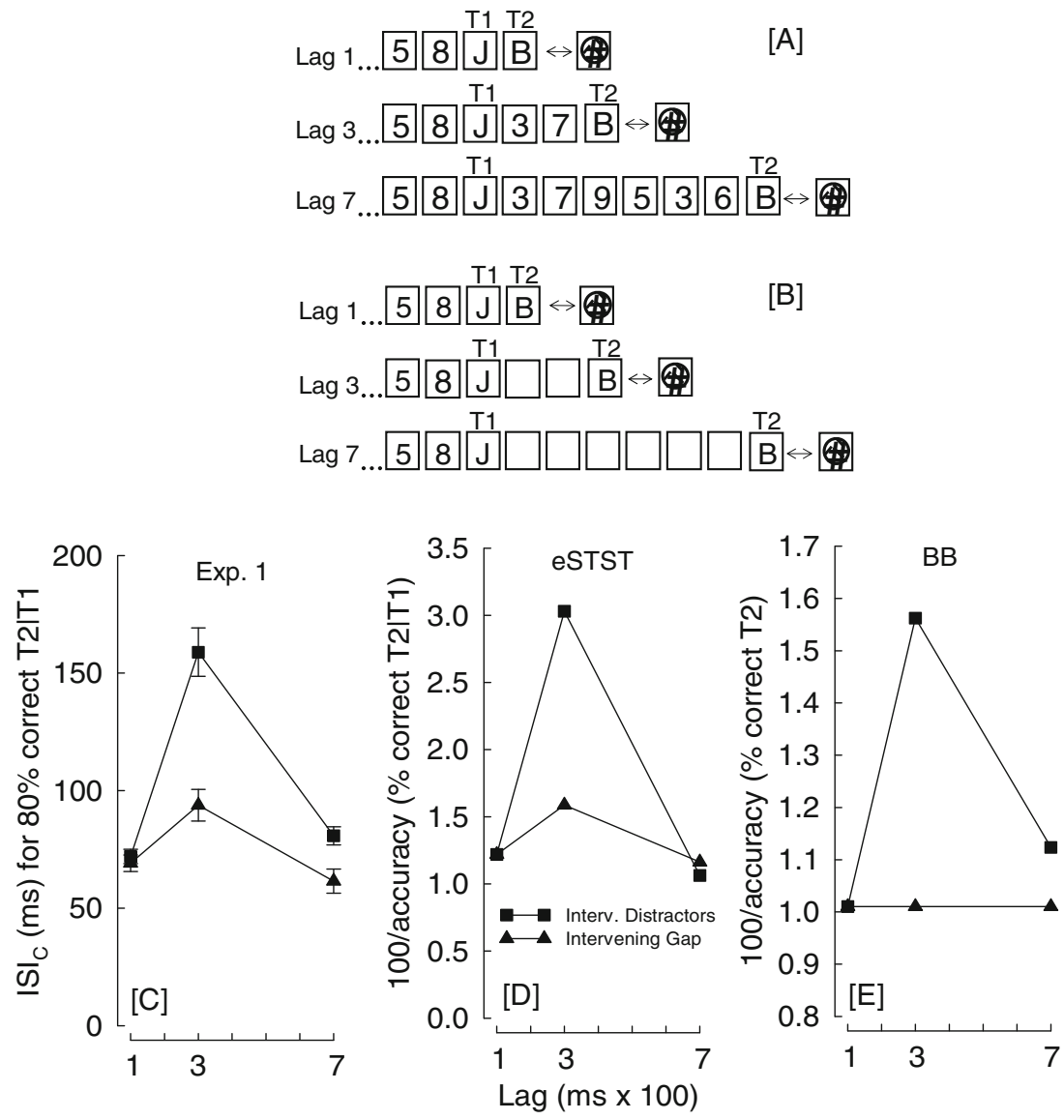
displays were viewed from a distance of approximately $60 \mathrm{~cm}$. At the beginning of each trial, a small fixation cross was presented in the center of the screen. Participants initiated each trial by pressing the space bar, at which point the fixation cross disappeared and the RSVP sequence began.

The number of distractors preceding T1 was determined randomly on each trial and varied between five and eight, inclusive. On any given trial, the distractors were selected randomly, with replacement, from the set of digits 2-9, with the constraint that the selected digit was not one of the two preceding items. In the intervening-distractors condition, distractors were displayed throughout the intertarget interval (Fig. 1a). In the intervening-gap condition, the intertarget distractors were replaced with blank frames of corresponding duration (Fig. 1b).

With the exception of the mask, which was displayed for $400 \mathrm{~ms}$, each item in the RSVP stream remained on the screen for approximately $7 \mathrm{~ms}$ and was followed by a 93-ms ISI during which the screen was blank, yielding a presentation rate of 10 items/s. The ISI between T2 and the mask was governed by PEST to yield the $\mathrm{ISI}_{\mathrm{C}}$ at which T2 could be identified on approximately $80 \%$ of the trials. To arrive at ISI $_{C}$, the T2-mask ISI was varied dynamically by PEST as follows. The ISI was reduced on trials in which response accuracy exceeded the $80 \%$ criterial level and was increased when accuracy was below that level. A Wald (1947) sequential likelihood-ratio test determined whether the accuracy of the immediately preceding run of responses was greater than or less than $80 \%$. The Wald routine was called only on trials on which $\mathrm{T} 1$ had been identified correctly. The PEST end-run consisted of 12 trials after three reversals in the direction of adjustment of the ISI had been recorded.

At the end of each trial, the participants were prompted to identify the targets by pressing the corresponding keys on the keyboard. They were instructed to report the letters in any order and to guess if uncertain. The fixation cross then reappeared, indicating readiness for the next trial.

Each participant took part in one session, consisting of three blocks of trials. In each of the blocks, there were three independent and randomly intermixed PEST runs, one for each of the three lags. Thus, participants could not anticipate the intertrial lag on any given trial. The assumption was made that the criterial level of performance had been approximated after three reversals in the direction of adjustment of the ISI. A separate estimate of $\mathrm{ISI}_{\mathrm{C}}$ for each lag was obtained in each block by averaging the T2-mask ISIs over the last 12 trials following the third PEST reversal. The final score $\left(\mathrm{ISI}_{\mathrm{C}}\right)$ was the median of the three $\mathrm{ISI}_{\mathrm{C}}$ estimates.

\section{Results and discussion}

The percentages of correct T1 identification in the interveningdistractors condition were $92 \%, 90 \%$, and $90 \%$ for lags 1, 3, and 7 , respectively. The corresponding percentages in the intervening-gap condition were $89 \%, 96 \%$, and $96 \%$. In all the present experiments, the percentages of correct T1 identifications were based on the 12 trials in the PEST end-run. The ISI $_{\mathrm{C}}$ scores, averaged over participants, are illustrated in Fig. 1c, separately for the two groups. It should be noted that low $\mathrm{ISI}_{\mathrm{C}}$ scores correspond to high $\mathrm{T} 2$ accuracy scores in conventional $\mathrm{AB}$ studies. Higher $\mathrm{ISI}_{\mathrm{C}}$ scores indicate that a longer mask-free interval is required to identify $\mathrm{T} 2$ to the PEST criterial level. Thus, higher $\mathrm{ISI}_{\mathrm{C}}$ scores reflect poorer performance (slower rate of processing) than lower ISI $_{C}$ scores. This, of course, is the opposite pattern to that obtained with accuracy as the dependent measure.

A 2 (condition) $\times 3$ (lag) mixed-factors ANOVA performed on the data in Fig. 1c revealed significant effects of condition, $F(1,26)=20.08, p<.001, M S E=886.98$, and lag, $F(2,52)=$ 93.23, $p<.001, M S E=308.59$. The interaction effect was also significant, $F(2,52)=23.60, p<.001, M S E=308.59$. Planned comparisons between the means for lags 3 and 7 revealed that an $\mathrm{AB}$ was obtained in both the intervening-distractors condition, $t(13)=8.86, p<.001$, and the intervening-gap condition, $t(13)=8.30, p<.001$. Coupled with the evidence in Fig. 1c, the significant interaction effect indicates that the magnitude of the AB was greater when the two targets were separated by intervening distractors than by intervening blanks. This was confirmed in a separate analysis in which the magnitude of the $\mathrm{AB}$ deficits (lag 3 minus lag 7) obtained in the two conditions were compared directly with one another, $t(26)=4.76, p<.001$.

The finding that the $\mathrm{AB}$ was in evidence with intervening distractors replicates conventional $\mathrm{AB}$ studies and is consistent with hypotheses stemming from both T1-based and distractor-based theories. The finding that an AB occurs without intervening distractors replicates the findings of Nieuwenstein, Potter, and Theeuwes (2009) with accuracy as the dependent measure. This pattern of results is consistent with T1-based models, which predict that $\mathrm{T} 1$ processing is sufficient for producing the $\mathrm{AB}$, but seemingly not with distractor-based theories, which hypothesize that intervening distractors are necessary for the AB.

The magnitude of the AB was considerably greater when the intertarget interval contained distractors than when it was blank (Fig. 1c). This result can be interpreted in at least two ways. First, it is possible that intervening distractors are sufficient, although not necessary, for producing the AB. On this option, the fact that an $\mathrm{AB}$ occurs in the absence of intervening distractors does not rule out the possibility that, when present, distractors play a role in eliciting the $\mathrm{AB}$. Second, it could be that, while not causing the $A B$, intervening distractors modulate its magnitude through backward masking of T1. Namely, on the twin assumptions that $\mathrm{T} 1$ difficulty modulates the magnitude of the $\mathrm{AB}$ and that masking increases $\mathrm{T} 1$ difficulty, masking of $\mathrm{T} 1$ by 
trailing distractors should increase the magnitude of the $\mathrm{AB}$. We return to this issue in Experiment 4.

Computer simulations of the eSTST model are compared with the results of the present experiments in Fig. 1d. The simulated results were obtained using the same model and parameters as were used by Wyble et al. (2009). In the eSTST simulations illustrated in Fig. 1d, the stimulus duration was set at $10 \mathrm{~ms}$, followed by an ISI of $90 \mathrm{~ms}$, similar to the actual experiment. In the eSTST model, distractors are prevented from reaching deeper levels of processing and, thus, cannot affect attention directly. Distractors are said to modulate the magnitude of the $\mathrm{AB}$ only indirectly by masking preceding targets. In the simulations, the effect of a temporal gap is implemented by removing this masking at appropriate points in the RSVP stream.

In contrast, in the B\&B simulations illustrated in Fig. 1e (Olivers \& Meeter, 2008), the stimulus duration was set at $100 \mathrm{~ms}$, with no ISI between successive items. ${ }^{1}$ Another difference between the two simulations is that the eSTST model simulated the results for $\mathrm{T} 2$ given that $\mathrm{T} 1$ was identified correctly, as was the case for the empirical results in Fig. 1c. Because the B\&B model has no such provision, the simulations represent the percentage of correct $\mathrm{T} 2$ responses, regardless of $\mathrm{T} 1$ accuracy.

The output of both simulations is expressed in terms of identification accuracy. In contrast, the empirical results were expressed in terms of ISI $_{\mathrm{C}}$. In order to make the shape of the simulated functions more similar to the shape of the empirical functions for ease of comparison, the simulated results were plotted as reciprocals (i.e., 100/accuracy). The range of values on the ordinates of Fig. 1d, e was chosen so as to maximize the visual similarity with the empirical functions in Fig. 1c. No parameters from either model were adjusted to fit the data apart from scaling of the vertical axis in these figures.

The eSTST simulations (Fig. 1d) provided a remarkably good prediction of the empirical data. ABs were obtained in both conditions, with the magnitude of the effect being larger in the intervening-distractors than in the interveninggap condition. The B\&B model (Fig. 1e) also revealed a large $\mathrm{AB}$ in the intervening-distractors condition but seemingly no $\mathrm{AB}$ in the intervening-gap condition.

It is possible that a ceiling constraint in the simulations may have prevented an $\mathrm{AB}$ from appearing in the $\mathrm{B} \& \mathrm{~B}$ simulations of the intervening-gap condition. Namely, when expressed in

\footnotetext{
${ }^{1}$ The B\&B model can simulate RSVP streams with blank ISIs. However, when we specified a 10-ms stimulus duration followed by a 90 ms blank, there were several aberrant features of the simulation. First, T1 accuracy at lags 1, 3, and 7 was $4 \%, 41 \%$, and $41 \%$. Second, the $\mathrm{AB}$ magnitude was excessively deep at lag 3 in the intervening condition, with accuracy of $0 \%$. Therefore, we opted to use a 0 -ms ISI so that $\mathrm{T} 1$ and $\mathrm{T} 2$ accuracy scores would be closer to the observed values.
}

terms of identification accuracy, the B\&B simulations show that accuracy in the intervening-gap condition was near ceiling ( $99 \%$ at all three lags). However, while not revealing an $\mathrm{AB}$ with accuracy as the response measure, the $\mathrm{B} \& \mathrm{~B}$ model does produce an $\mathrm{AB}$ when the response measure is the time taken for T2 to reach a given level of accuracy. For example, the modeled times for reaching an accuracy of $80 \%$ are 16 , 20 , and $16 \mathrm{~ms}$, at lags 1,3 , and 7 , respectively. ${ }^{2}$ Thus, both models produce some degree of $\mathrm{AB}$ even with an intervening blank. This is apparent in the accuracy data of the eSTST model and, with a more sensitive measure, similar to PEST, in the BB simulations.

The next two experiments were designed to test two possible ways in which the blank intertarget interval could have disrupted attention. In Experiment 2, we examined the option that the lack of visual stimulation during the intertarget interval in the intervening-gap condition in Experiment 1 may have caused attention to wander. In Experiment 3, we asked whether the intertarget gap might have come as a surprise to participants, thereby disrupting attention.

\section{Experiment 2}

Experiment 2 was designed to explore the possibility raised by Nieuwenstein, Potter, and Theeuwes (2009) that some extraneous factor related to the lack of visual stimulation during the intertarget gap played a causal role in the $\mathrm{AB}$ obtained with no intertarget distractors. Nieuwenstein et al. did not advance a reason why lack of visual stimulation could disrupt attention, thus leading to an AB. One possibility is that because there was nothing to hold attention to the location of the RSVP stream and because there were no intervening items that required processing, attention may have diffused or wandered, either in external space or inward to other mental processes (e.g., Smallwood \& Schooler, 2006), causing attention to be unavailable upon the presentation of $\mathrm{T} 2$.

The possibility of attention wandering during the intertarget gap is consistent with the finding of Nieuwenstein, Potter, and Theeuwes (2009, Experiment 2) that an AB-like deficit occurs when a single target is preceded by a gap in the RSVP stream of distractors (see also Lagroix, Spalek, \& Di Lollo, 2011). Although this AB was interpreted as arising from the inadvertent encoding of the distractor preceding the gap, it is also consistent with the option that a gap in the RSVP stream is sufficient for producing an AB. Evidence pertinent to this issue has been reported by Nieuwenstein, Van der Burg, et al. (2009, Experiment 2) who employed a "skeletal" $\mathrm{AB}$ paradigm consisting only of $\mathrm{T} 1, \mathrm{~T} 2$, and the T2 mask, without any leading or intervening distractors. The

\footnotetext{
${ }^{2}$ We thank Chris Olivers for running this simulation.
} 
objective was to determine whether a brief exposure of $\mathrm{T} 1$ was essential for obtaining an $\mathrm{AB}$ or whether an $\mathrm{AB}$ could be obtained also when T1 was presented throughout the intertarget interval. The finding of an $\mathrm{AB}$ in both conditions led to the conclusion that a brief exposure of T1 was not critical for the AB.

The long-exposure condition in Nieuwenstein, Van der Burg, et al.'s (2009) study is relevant to the objective of the present experiment. Namely, it is possible that the continued presence of $\mathrm{T} 1$ during the intertarget interval might have prevented attention from wandering. However, while entirely adequate for studying the relationship between T1 exposure duration and the $\mathrm{AB}$, the steady presence of $\mathrm{T} 1$ may have resulted in some habituation and, therefore, may not have prevented attention from wandering. For this reason, instead of presenting T1 steadily throughout the intertarget interval, we presented T1 repeatedly, separated by brief blanks. In such a segmented display, each presentation of T1 triggers an onset transient, which is likely to keep attention from wandering (Phillips \& Singer, 1974).

In brief, the present experiment had two main objectives. One was to determine whether it is the lack of visual stimulation during the intertarget interval that causes the $\mathrm{AB}$ in experiments in which a gap is inserted between the two targets. To this end, we presented the T1 letter repeatedly during the intertarget interval, as illustrated in Fig. 2 a. This ensured that visual stimulation was present while avoiding the presentation of distractors. The repeated presentation of T1, instead of some other visual stimulus such as random dots, was aimed at the second objective of Experiment 2-namely, to provide a further test between the two classes of theory. According to distractor-based theories, no AB should occur because, while the repeated presentation of $\mathrm{T} 1$ prevents attention from wandering, no distractors are displayed between the two targets. In contrast, because $\mathrm{T} 1$ still requires processing, an $\mathrm{AB}$ is predicted by T1-based theories. The magnitude of the AB, however, is expected to be relatively small because $\mathrm{T} 1$ is not masked by a different trailing stimulus and because $\mathrm{T} 1$ processing is facilitated by the repeated presentation.

\section{Method}

\section{Participants}

Eighteen undergraduate students participated for course credit. All reported normal or corrected-to-normal vision and were naiive as to the purpose of the experiment.
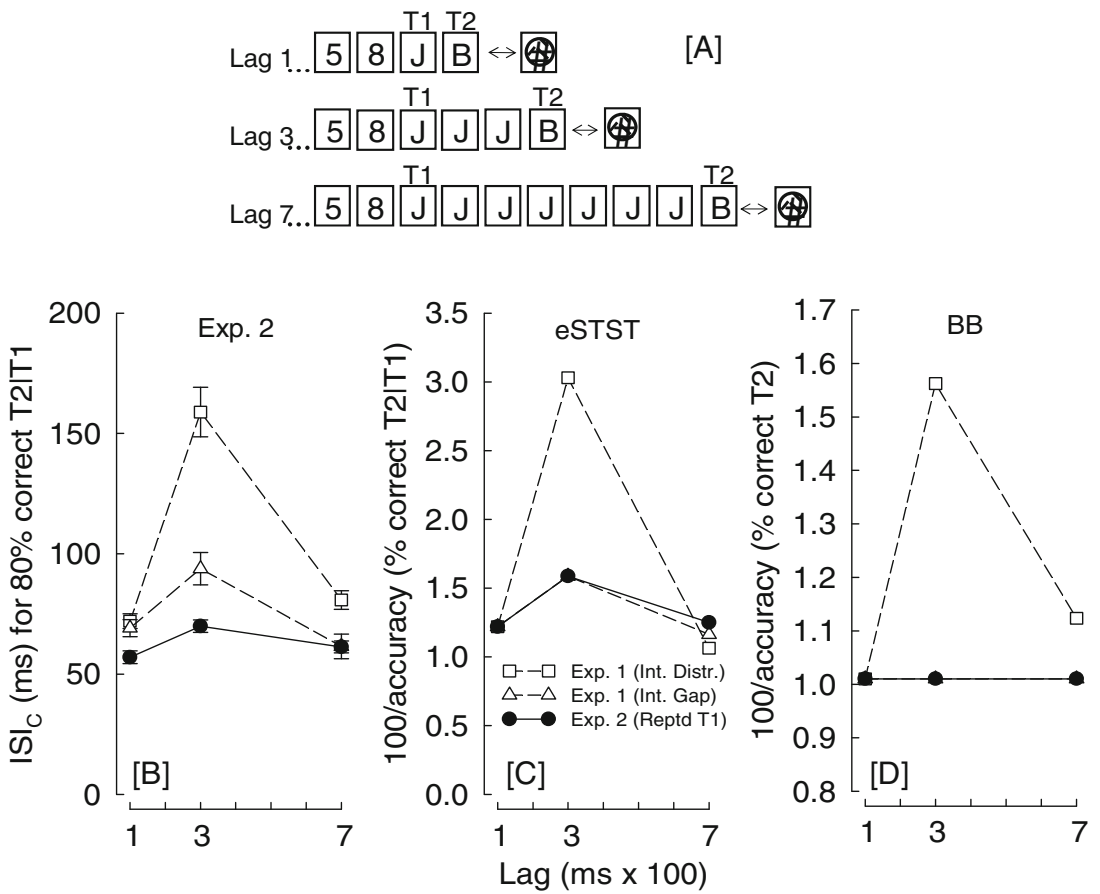

Fig. 2 a Schematic representation of the sequence of the events in Experiment 2. Each square represents one 7.14-ms frame, except for the mask, which was presented for $400 \mathrm{~ms}$. The small gap between each square represents a blank ISI of $93 \mathrm{~ms}$. The T2-mask ISI was governed by PEST, which converged on the critical ISI (ISI $\mathrm{C}_{\mathrm{C}}$ ) required for $80 \%$ correct $\mathrm{T} 2$ identifications. $\mathbf{b}$ Mean $\mathrm{ISI}_{\mathrm{C}}$ as a function of lag. The solid-line function illustrates the results of Experiment 2. The segmented-line functions illustrate the results of Experiment 1, included for ease of comparison. Int. = intervening; ISI = interstimulus interval; $\mathrm{ms}=$ milliseconds. Error bars indicate standard errors of the means. $\mathbf{c}$ and $\mathbf{d}$ Computer simulations from the eSTST and the B\&B models, respectively. The solid-line functions illustrate simulations for Experiment 2. The segmented-line functions illustrate the simulations for Experiment 1, included for ease of comparison 


\section{Design and procedure}

Apparatus, design, and procedures were the same as in the intervening-distractors condition in Experiment 1, with the exception that the distractors intervening between the two targets were replaced with repetitions of $\mathrm{T} 1$ (see Fig. 2a). That is, T1 was presented once at lag 1, three times at lag 3 , and seven times at lag 7 .

\section{Results and discussion}

The percentages of correct $\mathrm{T} 1$ identification were $92 \%$, $96 \%$, and $94 \%$ for lags 1, 3, and 7, respectively. The ISI scores, averaged over participants, are illustrated in solidline function in Fig. 2b. The segmented-line functions in Fig. $2 \mathrm{~b}$ represent the results of Experiment 1 and are reproduced here for ease of comparison. A one-way withinsubjects ANOVA performed on the ISI $_{\mathrm{C}}$ scores for Experiment 2 yielded a significant effect of lag, $F(2,34)=$ 20.85, $p<.001, M S E=37.48$. Planned comparisons between the means for lags 3 and 7 confirmed that a significant AB was obtained, $t(17)=4.56, p<.001$, albeit smaller than that obtained with a blank intertarget interval in Experiment 1, $t(30)=5.87, p<.001$. This is consistent with predictions from T1-based theories, which would claim that the repeated presentation of $\mathrm{T} 1$ should facilitate its processing, thereby reducing the magnitude of the $\mathrm{AB}$.

The relatively small but significant $A B$ observed in the present experiment indicates that an $\mathrm{AB}$ can occur in the absence of intervening distractors even when there is visual stimulation throughout the intertarget interval. This suggests that it was not the lack of visual stimulation that caused an $\mathrm{AB}$ in experiments in which there was an intertarget gap (e.g., Experiment 1, intervening-gap condition; Nieuwenstein, Potter, \& Theeuwes, 2009; Nieuwenstein, Van der Burg, et al., 2009). In addition, it provides further evidence against distractor-based theories, which state that the presence of a distracting event intervening between the two targets is necessary for the $\mathrm{AB}$. In contrast, this finding is entirely consistent with T1-based models, because the requirement to process $\mathrm{T} 1$ was sufficient for producing an $\mathrm{AB}$.

Computer simulations of the eSTST and the B\&B models are illustrated in Fig. 2c, d, respectively. The eSTST model correctly predicts an $\mathrm{AB}$ in the present experiment, but fails to reproduce its reduced magnitude relative to the intervening-gap condition of Experiment 1 . To be sure, the simulated magnitude of the $\mathrm{AB}$ (lag 7 minus lag 3) was smaller in Experiment 2 than in the intervening-gap condition of Experiment 1, but that was due to worse performance at lag 7 rather than to better performance at lag 3 .

The $\mathrm{B} \& \mathrm{~B}$ simulations (Fig. 2d) reveal no $\mathrm{AB}$ at all. Because of the possibility, noted above, that a ceiling constraint might have prevented an $\mathrm{AB}$ from appearing, we ran a $B \& B$ simulation with a stimulus duration of $10 \mathrm{~ms}$, which again revealed no $A B$. Indeed a reverse- $A B$ was in evidence: The percentages of simulated correct $\mathrm{T} 2$ responses were 96, 95, and 93 for lags 1, 3, and 7, respectively, illustrating an impairment rather than an enhancement from lag 3 to lag 7. As a further check on this result, and as was done in Experiment 1, we repeated the present simulations with a stimulus duration of $100 \mathrm{~ms}$ and estimated the time taken for T2 to reach an accuracy of $80 \%$. The simulations showed that the $80 \%$ level was reached after 16, 17, and $20 \mathrm{~ms}$ at lags 1,3 , and 7 , respectively, again revealing a reverse- $\mathrm{AB}$. Thus, the $\mathrm{B} \& \mathrm{~B}$ model is unable to account for the $\mathrm{AB}$ obtained when $\mathrm{T} 1$ is repeated throughout the intertarget interval.

\section{Experiment 3}

Experiment 3 was designed to explore ways in which T1based models might account for the finding that an $\mathrm{AB}$ occurs when the intertarget distractors are replaced by blank frames. The TLC model might postulate that the relatively long blank intertarget interval (e.g., the 293-ms gap at lag 3 in Experiment 1) might be perceived as an unexpected deviation from the 100-ms rhythm established in the leading RSVP stream (93-ms interitem gaps). As such, the long intertarget gap at lag 3 might come as a "surprise," thereby disrupting attention and causing an AB. This possibility was not addressed in Experiment 2, in which $\mathrm{T} 1$ was presented repeatedly throughout the intertarget interval. While this maintained the presentation rhythm, the repetitions themselves might have come as a surprise, since, in the leading stream, no items were ever repeated sequentially. Like an extended gap, this unexpected repetition may have disrupted attention, thereby leading to an $\mathrm{AB}$.

In the $\mathrm{B} \& \mathrm{~B}$ model, the blank intertarget interval is assigned an inhibitory valence. The rationale for the inhibition is much the same as that described above for the TLC model (C. N. L. Olivers, personal communication, April 2012). Within the B\&B model, not all blanks are regarded as inhibitory. For example, the brief ISIs between successive items in the RSVP stream are modeled as decaying activation with no inhibitory valence. Intertarget gaps, such as those in the intervening-gap condition in Experiment 1, are said to be disruptive because they constitute deviations from the rhythm established in the leading RSVP stream. Such deviations come as "surprises" that disrupt attention, leading to an $\mathrm{AB}$.

In the present experiment, the presentation rate of the leading stream was adjusted by replacing the $93-\mathrm{ms}$ interitem gap with a 293-ms interitem gap such that the interitem gaps in the leading stream matched the intertarget gap at lag 3 . If the deficit observed at lag 3 in Experiment 1 (intervening-gap 
condition) was due to the unexpectedly long intertarget gap, no such deficit should be observed in the present experiment. This is because the duration of the gap at lag 3 does not deviate from the rhythm established in the leading RSVP stream. It needs to be noted that the long gap preceding T2 at lag 7 would not be expected to produce a deficit in T2 identification, because, consistent with distractor-based models, the additional duration of the gap would allow the system to recover from the initial "surprise."

The experimental design employed in a study by Nieuwenstein, Van der Burg, et al. (2009, Experiment 1) bears some similarity to the present design in that there were no distractors intervening between the two targets. The key difference from the present work was the absence of a leading RSVP stream of distractors. This was done with the intent of determining whether an $\mathrm{AB}$ can be obtained in the total absence of distractors. To this end, the RSVP stream consisted of only three items: T1, T2, and a mask after T2. Notably, the absence of a leading RSVP stream did not allow for the establishment of a rhythm-based expectation. The Nieuwenstein, Van der Burg, et al. (2009) study clearly established that an AB can be obtained in the total absence of distractors. However, it did not rule out the option that violation of expectation may cause the $\mathrm{AB}$ when no distractors intervene between the two targets and the duration of the intertarget gap comes as a surprise. This is because the observers may well have approached the task with some kind of expectation, but the nature of that expectation was indeterminate. In contrast, in the present study, we manipulated the observers' expectation explicitly by establishing a rhythm during the leading RSVP stream.

It should be mentioned, at least in passing, that T1-based models - notably, eSTST - would predict an AB in the present experiment on the grounds that the $\mathrm{AB}$ is contingent on the requirement to process T1. And that requirement was the same in the present experiment as in Experiment 1.

\section{Method}

\section{Participants}

Sixteen undergraduate students participated for course credit. All reported normal or corrected-to-normal vision and were naïve as to the purpose of the experiment.

\section{Design and procedure}

The apparatus, design, and procedures were the same as those in the intervening-gap condition in Experiment 1, with the exception that the 93-ms blank ISI between the leading (i.e., pre-T1) distractors was replaced with a 293-ms blank ISI. Consequently, in the leading stream, a new item appeared every $300 \mathrm{~ms}$ (see Fig. 3a), rather than every $100 \mathrm{~ms}$, as in Experiment 1. The blank intertarget gap was the same as in Experiment 1 (intervening-gap condition): 93, 293, and $693 \mathrm{~ms}$, for lags 1, 3, and 7, respectively.

Results and discussion

The percentages of correct T1 identification were $88 \%$, $97 \%$, and $96 \%$ for lags 1,3 , and 7 , respectively. The ISI $_{\mathrm{C}}$ scores, averaged over participants, are illustrated by the solid-line function in Fig. 3b. A one-way within-subjects ANOVA performed on the ISI $_{\mathrm{C}}$ scores yielded a significant effect of lag, $F(2,30)=13.08, p<.001, M S E=210.42$. A planned comparison between the means for lags 3 and 7 confirmed that a significant $\mathrm{AB}$ was obtained, $t(15)=3.60$, $p<.001$.

The finding of a significant $\mathrm{AB}$ is not consistent with the TLC predictions outlined above. Indeed, a comparison between the results of the present experiment and those of the intervening-gap condition in Experiment 1 shows that expectations based on the rhythm established during the leading RSVP stream played no role in determining any of the results in Fig. 3b. On the expectation hypothesis, the AB obtained in the intervening-gap condition of Experiment 1 is explained as follows: The 100-ms presentation rate in the leading stream established an expectation that a new item would be presented every $100 \mathrm{~ms}$. The long 300-ms SOA at lag 3 then came as a surprise that disrupted attention and interfered with the processing of $\mathrm{T} 2$. No disconfirmation of expectation occurred at lag 1 because T2 appeared at the expected 100-ms SOA. Performance at lag 7 was not affected because, as was noted above, the additional duration of the intertarget gap allowed the system to recover from the initial surprise. With respect to lags 1 and 3, the converse contingencies apply in the present experiment, in which the initial RSVP stream established an expectation that a new item would be presented every $300 \mathrm{~ms}$. In this case, T2 should have come as a surprise at lag 1, but not at lag 3. Thus, a deficit should have occurred at lag 1, but not at lag 3 .

On this reasoning, a comparison between the results of the intervening-gap condition in Experiment 1, and the results of the present experiment should reveal a lag $\times$ experiment interaction effect. The functions in Fig. $3 \mathrm{~b}$ show no evidence of such an interaction. This was confirmed in a 2 (Experiment, 1 or 3 ) $\times 3$ (lag, 1, 3, or 7) mixed-design ANOVA in which the results of the intervening-gap condition in Experiment 1 were analyzed along with the results of the present experiment. The analysis revealed significant effects of experiment, $F(1,28)=4.73, p=.038, M S E=$ 577.17 , and lag, $F(2,56)=36.22, p<.001, M S E=184.08$. Notably, the interaction effect was not significant $(F<1)$.

On the face of it, the occurrence of an $\mathrm{AB}$ in the present experiment is entirely consistent with predictions from the eSTST model (Fig. 3c), in which the AB is seen as critically 

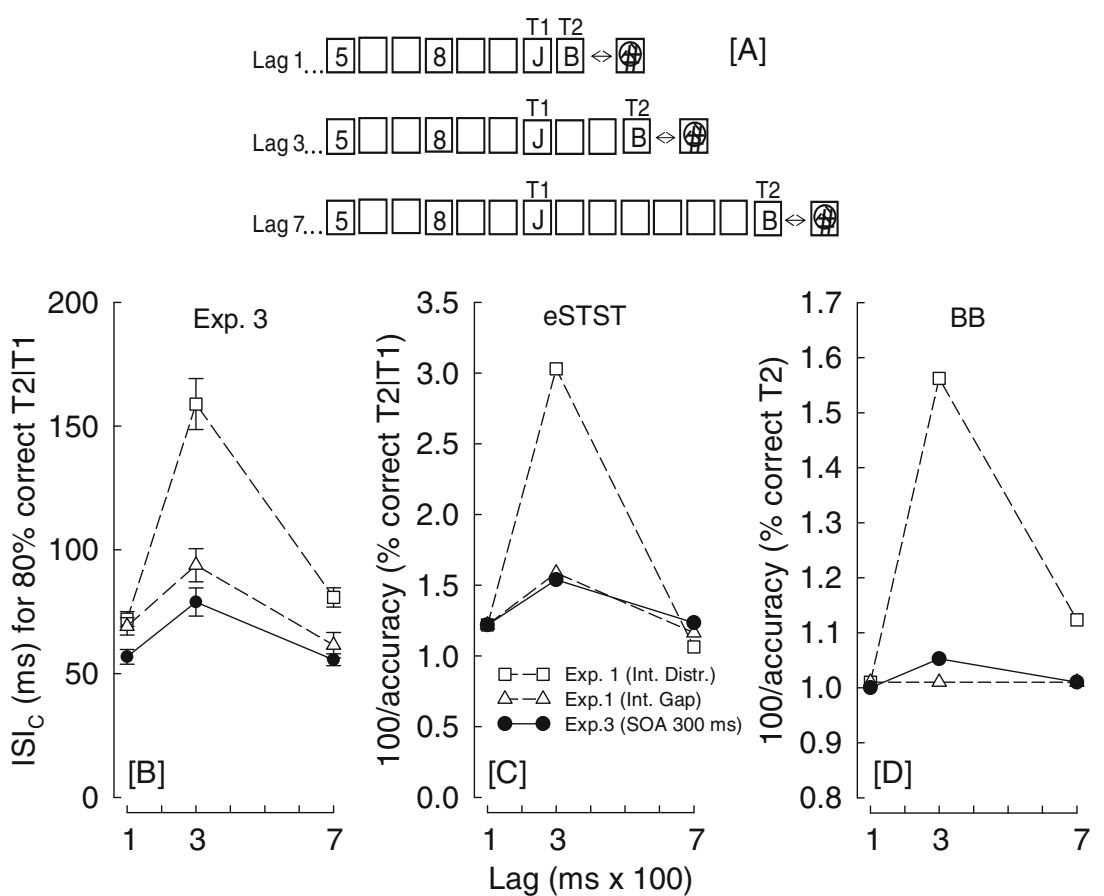

Fig. 3 a Schematic representation of the sequence of events in the in Experiment 3. Each square represents one 7.14-ms frame, except for the mask, which was presented for $400 \mathrm{~ms}$. The small gap between each square represents a blank ISI of $93 \mathrm{~ms}$. The T2-mask ISI was governed by PEST, which converged on the critical ISI (ISI $\mathrm{C}_{\mathrm{C}}$ ) required for $80 \%$ correct T2 identifications. b Mean ISI $_{C}$ as a function of lag. The solid-line function illustrates the results of Experiment 3. The segmented-line functions illustrate the results of Experiment 1,

dependent on $\mathrm{T} 1$ processing. This result is also simulated successfully by the B\&B model (Fig. 3d). On the other hand, the rationale for attributing a negative valence to the intertarget gap is the same in the B\&B model as in the TLC model - namely, the "surprising" deviation of the intertarget gap duration from the rhythm established during the leading RSVP stream. Thus, it seems that the B\&B model predicts the results, but for the wrong reason. Clearly, the $\mathrm{B} \& \mathrm{~B}$ model needs to put forth a better rationale for modeling the intertarget gap. In brief, the results of the present experiment provide further evidence against distractor-based theories, as presently formulated, and suggest that, consistent with T1-based theories, T1 encoding alone is sufficient for the occurrence of an $\mathrm{AB}$.

\section{Experiment 4}

Considered collectively, the results of Experiments 1-3 are not consistent with predictions from distractor-based theories and lend strong support to the claim of T1-based theories that $\mathrm{T} 1$ processing alone is sufficient for producing the $\mathrm{AB}$. While not a primary determinant of the $\mathrm{AB}$, however, intervening distractors do play a role in determining its included for ease of comparison. Int. = intervening; ISI = interstimulus interval; $\mathrm{ms}=$ milliseconds; $\mathrm{SOA}=$ stimulus onset asynchrony. Error bars indicate standard errors of the means. c and d Computer simulations from the eSTST and the B\&B models, respectively. The solidline functions illustrate simulations for Experiment 3. The segmentedline functions illustrate the simulations for Experiment 1, included for ease of comparison

magnitude. The results of Experiment 1 (Fig. 1c) show that the $\mathrm{AB}$ was substantially larger when the intertarget interval contained distractors than when it was blank. A similar pattern of results has been reported by Nieuwenstein, Potter, and Theeuwes (2009), who attributed the larger AB to increased difficulty of $\mathrm{T} 1$ processing due to backwardmasking of T1 when the ensuing item was a distractor.

The question now arises as to whether masking of T1 can account for the entire difference in the magnitude of the $\mathrm{AB}$ obtained with and without intervening distractors, as predicted by eSTST, or whether the distractors also caused some disruption in input control, as proposed by distractor-based theories. Experiment 4 was designed to determine whether disruption of input control contributed to the larger $\mathrm{AB}$ obtained in the intervening-distractors than in the intervening-gap condition in Experiment 1. There were two conditions: distractor at $T 1+1$ (henceforth, $T 1+1)$ and distractor at $T 1+2$ (henceforth, $T 1+2$ ). The RSVP stream in both conditions was the same as in the intervening-gap condition in Experiment 1, with the following exceptions: In the $\mathrm{T} 1+1$ condition, the blank frame directly following $\mathrm{T} 1$ was replaced with a distractor, and in the $\mathrm{T} 1+2$ condition, the second blank frame following $\mathrm{T} 1$ was replaced with a distractor (see Fig. 4a, b ). 

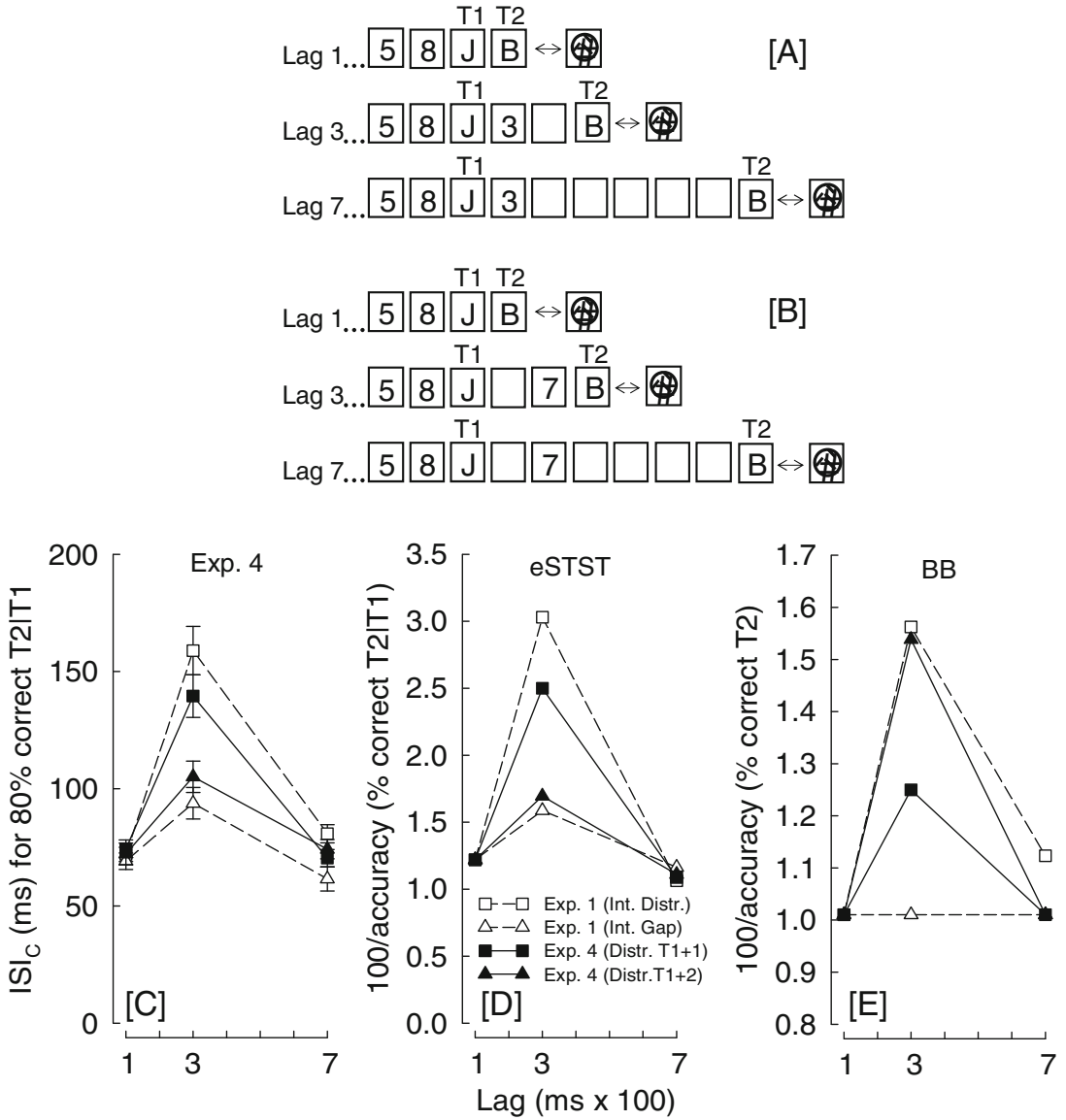

Fig. 4 a and $\mathbf{b}$ Schematic representation of the sequence of events in the in the $\mathrm{T} 1+1$ and the $\mathrm{T} 1+2$ conditions, respectively, in Experiment 4. Each square represents one 7.14-ms frame, except for the mask, which was presented for $400 \mathrm{~ms}$. The small gap between each square represents a blank ISI of $93 \mathrm{~ms}$. The T2-mask ISI was governed by PEST, which converged on the critical ISI $\left(\mathrm{ISI}_{\mathrm{C}}\right.$ ) required for $80 \%$ correct $\mathrm{T} 2$ identifications. $\mathbf{c}$ Mean $\mathrm{ISI}_{\mathrm{C}}$ as a function of lag. The solidline functions illustrate the results of Experiment 4. The segmented-

On the hypothesis that in Experiment 1, the larger $\mathrm{AB}$ obtained with intervening distractors was due to masking of $\mathrm{T} 1$, we expected the results of the $\mathrm{T} 1+1$ condition to match those of the intervening-distractors condition in Experiment 1 (Fig. 1c). This expectation was based on the finding that the magnitude of the $\mathrm{AB}$ remains the same whether $\mathrm{T} 1$ is followed by only one distractor or by many (Giesbrecht \& Di Lollo, 1998). In a similar vein, we expected the results of the $\mathrm{T} 1+2$ condition to match those of the intervening-gap condition in Experiment 1. This was based on the finding that a single letter (i.e., T1) is not masked if the trailing mask is presented at an SOA greater than about $150 \mathrm{~ms}$ (Spencer \& Shuntich, 1970). On the other hand, to the extent that disruption of input control contributes to the magnitude of the $\mathrm{AB}$, a larger $\mathrm{AB}$ should be obtained in the $\mathrm{T} 1+2$ condition (in which a distractor is presented directly before T2) than in the intervening-gap condition in Experiment 1. line functions illustrate the results of Experiment 1, included for ease of comparison. Int. $=$ intervening; ISI $=$ interstimulus interval; $\mathrm{ms}=$ milliseconds; Distr. $=$ distractor. Error bars indicate standard errors of the means. $\mathbf{d}$ and $\mathbf{e}$ Computer simulations from the eSTST and the $\mathrm{B} \& \mathrm{~B}$ models, respectively. The solid-line functions illustrate simulations for Experiment 4 . The segmented-line functions illustrate the simulations for Experiment 1, included for ease of comparison

Method

\section{Participants}

Thirty-four undergraduate students participated for course credit. All reported normal or corrected-to-normal vision and were naïve as to the purpose of the experiment. They were randomly assigned to two groups of 17 participants each: the $T 1+1$ and the $T 1+2$ groups.

\section{Design and procedure}

The apparatus, design, and procedures were the same as those in the intervening-gap condition in Experiment 1, with the exceptions noted above. The RSVP sequences in the $\mathrm{T} 1+1$ and the $\mathrm{T} 1+2$ conditions are illustrated in Fig. $4 a, b$, respectively. 


\section{Results and discussion}

The percentages of correct $\mathrm{T} 1$ identification in the $\mathrm{T} 1+1$ condition were $91 \%, 90 \%$, and $90 \%$ for lags 1,3 , and 7 , respectively. The corresponding percentages in the $\mathrm{T} 1+2$ condition were $87 \%, 93 \%$, and $89 \%$. The ISI $_{\mathrm{C}}$ scores, averaged over participants, are illustrated in Fig. 4c, separately for the two groups. A 2 (condition) $\times 3$ (lag) mixedfactors ANOVA performed on the data illustrated by the solid lines in Fig. 4c revealed significant effects of lag, $F$ $(2,64)=69.13, p<.001, M S E=401.54$, and a condition $\times$ lag interaction, $F(2,64)=9.01, p<.001, M S E=401.54$. The effect of condition was not significant, $F(1,32)=3.60$, $p=.067, M S E=836.01$. Planned comparisons between the means for lags 3 and 7 revealed that an $\mathrm{AB}$ was obtained in both the $\mathrm{T} 1+1$ distractor condition, $t(16)=8.07, p<.001$, and the $\mathrm{T} 1+2$ distractor condition, $t(16)=5.44, p<.001$. Coupled with the evidence in Fig. 4c (solid lines), the significant interaction effect indicates that the magnitude of the $\mathrm{AB}$ was greater in the $\mathrm{T} 1+1$ condition than in the $\mathrm{T} 1+2$ condition. This was confirmed in a separate analysis in which the magnitude of the $\mathrm{AB}$ (lag 7 minus lag 3) obtained in the two conditions were compared directly with one another, $t(32)=3.69, p<.001$.

The question of principal interest was whether the magnitude of the ABs obtained in the present experiment differed from those in Experiment 1. This involves two separate comparisons: (1) the $\mathrm{AB}$ (lag 7 minus lag 3) in the intervening-distractors condition in Experiment 1 versus the $\mathrm{AB}$ in the $\mathrm{T} 1+1$ condition in the present experiment and (2) the $A B$ in the intervening-gap condition in Experiment 1 versus the $A B$ in the $T 1+2$ condition in the present experiment (see Fig. 4c). Neither comparison was statistically significant, $t(29)=0.73, p=.472$, and $t(29)=0.16, p=$ .873 , for (1) and (2), respectively.

Simulations from the eSTST and B\&B models are illustrated in Fig. 4d, e, respectively. The eSTST simulations provided a remarkably close prediction of the empirical results. In contrast, the $\mathrm{B} \& \mathrm{~B}$ model predicted a larger $\mathrm{AB}$ in the $\mathrm{T} 1+2$ than in the $\mathrm{T} 1+1$ condition, which is the reverse of the empirical findings. Clearly, the B\&B model is in need of modification in order to account for the results of the present experiment.

The finding that the magnitude of the $\mathrm{AB}$ in the $\mathrm{T} 1+2$ condition did not differ from that in the intervening-gap condition of Experiment 1indicates that the intervening distractor in the $\mathrm{T} 1+2$ condition did not affect the magnitude of the $\mathrm{AB}$ either by masking $\mathrm{T} 1$ or by disrupting input control. As was noted above, the absence of masking was to be expected on the grounds that backward masking of a single letter does not occur at SOAs beyond about $150 \mathrm{~ms}$ (Spencer \& Shuntich, 1970). The absence of interference with input control, however, is inconsistent with the idea that the larger $\mathrm{AB}$ seen in the intervening-distractors condition of Experiment 1 was due, at least in part, to disruption of input control by intervening distractors. Instead, this finding strongly suggests that the principal role of the intervening distractors in modulating the magnitude of the $A B$ is through backward masking of T1. One way in which masking of $\mathrm{T} 1$ can result in a larger deficit of $\mathrm{T} 2$ identification is by increasing the time required to process $\mathrm{T} 1$. This causes the processing of $\mathrm{T} 2$ to be delayed by a period during which its representation is vulnerable to decay and overwriting, with corresponding impairment in identification accuracy.

On this hypothesis, one would expect identification accuracy of T1 to be lower when it is masked than when it is not masked. An analysis of the results, however, revealed that identification accuracy of T1, averaged across lags 3 and 7, was $90 \%$ and $91 \%$ in the $\mathrm{T} 1+1$ and the $\mathrm{T} 1+2$ conditions, respectively (lag 1 was not included because the stimulus sequence was identical in the two conditions). Although this is seemingly inconsistent with the T1masking hypothesis, the possibility needs to be considered that the $100 \%$ limit of the response scale imposed a ceiling, which prevented a difference between the two conditions from coming into evidence. Namely, it is possible that, even though identification accuracy was the same in the two conditions, masking might have impaired other facets of target processing. For example, processing might have taken longer when the target was masked than when it was not masked.

This hypothesis was corroborated by the outcome of a subsidiary experiment, reported in the Appendix, in which the reaction time (RT) to a target that was followed by a mask was found to be longer than the RT to a target that was followed by a blank. This outcome buttresses the conclusion reached in the present experiment that the principal role of the intervening distractors in modulating the magnitude of the $\mathrm{AB}$ is through backward masking of $\mathrm{T} 1$. This conclusion is more clearly consistent with T1-based theories than with distractor-based theories.

\section{Summary and conclusions}

The present work addressed the distinction between two classes of theory of the AB: T1-based theories, which hold that $\mathrm{T} 1$ processing alone is sufficient to produce the $A B$, and distractor-based theories, in which the presence of at least one distractor following $\mathrm{T} 1$ is regarded as essential. On the face of it, distractor-based theories were disconfirmed by the finding that the $\mathrm{AB}$ occurs even in the absence of distractors intervening between the two targets (Nieuwenstein, Potter, \& Theeuwes, 2009). The principal objective of the present experiments was to explore ways in which that finding might be reconciled with distractor-based theories. Specifically, we 
aimed at identifying any factors associated with the intertarget blank that might bring about an AB.

In Experiment 1, a threshold-tracking procedure not subject to ceiling constraints (PEST) was used to replicate the findings of Nieuwenstein, Potter, and Theeuwes (2009). The dependent measure was the critical T2-mask ISI (ISI $\left.{ }_{C}\right)$ required to identify T2 on approximately $80 \%$ of the trials. The next two experiments were designed to test two possible ways in which the blank intertarget interval might have disrupted attention, leading to an AB. Experiment 2 examined the hypothesis that the lack of visual stimulation during the blank intertarget interval may have caused attention to wander. To this end, the blank intertarget frames were replaced with repeated presentations of $\mathrm{T} 1$. $\mathrm{An} \mathrm{AB}$ occurred, consistent with T1-based but not with distractorbased theories.

In Experiment 3, the presentation rate of the leading stream was adjusted by changing the duration of the interitem gap from 93 to $293 \mathrm{~ms}$ such that the interitem gaps in the leading stream matched the intertarget gap at lag 3 . An $\mathrm{AB}$ was again observed, confirming predictions from $\mathrm{T} 1$ based but not from distractor-based theories.

Experiment 4 was designed to explore the robust finding that the magnitude of the $\mathrm{AB}$ is greater when the intertarget interval contains distractors than when it is blank. This difference has been attributed to increased difficulty of T1processing caused by masking of $\mathrm{T} 1$ by the trailing distractors (Nieuwenstein, Potter, \& Theeuwes, 2009). We asked whether masking of $\mathrm{T} 1 \mathrm{can}$ account for the entire difference in the magnitude of the $\mathrm{AB}$ obtained with and without intervening distractors or whether the distractors also cause some disruption in input control, as proposed by distractorbased theories. There were two conditions: distractor at $T 1+1$, and distractor at $T 1+2$. The results, corroborated by the data in the Appendix, revealed no evidence for disruption of input control. Rather, they indicated that the only role of the intervening distractors in modulating the magnitude of the $\mathrm{AB}$ was through backward masking of $\mathrm{T} 1$. Computer simulations based on the eSTST (Wyble et al., 2009) and the B\&B (Olivers \& Meeter, 2008) models were performed for each of the four experiments. These simulations used the models in their original published form, without parameter adjustment apart from scaling of the vertical axis for visualization purposes. The eSTST simulations provided remarkably good predictions in every case. The B\&B simulations, on the other hand, had only partial success in predicting an $\mathrm{AB}$ in the intervening-gap condition of Experiment 1 and predicted no $\mathrm{AB}$ in Experiment 2, in which $\mathrm{ABs}$ were actually in evidence. The $\mathrm{B} \& \mathrm{~B}$ model correctly simulated an $\mathrm{AB}$ in Experiment 3, but for the wrong reason. Also, $\mathrm{B} \& \mathrm{~B}$ predicted the opposite pattern of results to that actually obtained in Experiment 4. This is not to say that the $\mathrm{B} \& \mathrm{~B}$ model cannot be revised to provide a suitable account of the results, but in its present form, it generates poor predictions of the empirical data.

As was noted in the foregoing, the T1-based class of theory encompasses a number of different individual theories, such as the two-stage (e.g., Chun \& Potter, 1995), bottleneck (e.g., Jolicoeur \& Dell'Acqua, 1998), and resource depletion (Ward et al., 1996) models. All these theories are capable of accounting for the results of the present work, since they all share the common tenet that the $\mathrm{AB}$ results from the requirement to process $\mathrm{T} 1$. They are not, however, equally capable of accounting for all of the findings in the $\mathrm{AB}$ literature. For example, two-stage, bottleneck, and resource-depletion models have all been challenged by the finding that an $\mathrm{AB}$ does not occur when multiple targets are presented in succession (e.g., Di Lollo et al., 2005; Kawahara et al., 2006; Olivers \& Meeter, 2008; Olivers et al., 2007; but see Dell'Acqua, Jolicoeur, Luria, \& Pluchino, 2009; Dux, Asplund, \& Marois, 2008, 2009, for an alternative interpretation, and Olivers, Hulleman, Spalek, Kawahara, \& Di Lollo, 2011; Olivers, Spalek, Kawahara, \& Di Lollo, 2009, for rebuttals).

An important point to consider is how T1-based accounts can be reconciled with the findings that the $\mathrm{AB}$ is abated by multiple sequential targets. Even lag-1 sparing would seem, at first glance, to contradict the notion of a $\mathrm{T} 1$-based $\mathrm{AB}$, since $\mathrm{T} 1$ is masked by $\mathrm{T} 2$. This apparent contradiction is resolved in the eSTST model by positing that attention is controlled not only by $\mathrm{T} 1$ processing, but also by a balance of competing influences, both excitatory and inhibitory. In the model, T1 processing always causes suppression of attention, but that suppression can be counteracted by excitation from targets that follow $\mathrm{T} 1$ in immediate succession (Wyble et al., 2009; Wyble et al., 2011). Thus, even though T1 is masked by an immediately trailing 2 and $\mathrm{T} 3$, a sequence of three consecutive targets keeps the attentional mechanism strongly active and abates the $\mathrm{AB}$.

In a recent review of the $\mathrm{AB}$ literature, Dux and Marois (2009) concluded that the eSTST model of Wyble et al. (2009) is capable of accounting for the largest number of empirical findings. That conclusion is buttressed by the present experiments, which collectively disconfirm predictions from distractor-based theories, while lending strong support to the claim that $\mathrm{T} 1$ processing alone is sufficient for the occurrence of the $\mathrm{AB}$.

Author Note This work was supported by Alexander Graham Bell Canada Graduate Scholarships from the Natural Sciences and Engineering Research Council of Canada (NSERC) to H.E.P.L. and A.J., by a Canadian Foundation for Innovation New Opportunities grant and a grant from the British Columbia Knowledge Development Fund to T.M.S., by Discovery Grants from NSERC to T.M.S. and V.D.L., and by Grant MH47432 from the National Institute of Mental Health to B.W. 


\section{Appendix}

The present experiment was designed to test the hypothesis that a response ceiling concealed a difference in $\mathrm{T} 1$ performance between the $\mathrm{T} 1+1$ and the $\mathrm{T} 1+2$ conditions in Experiment 4. There were two conditions, corresponding to the $\mathrm{T} 1+1$ and the $\mathrm{T} 1+2$ conditions in Experiment 4, except that the RSVP stream contained only one target. In the $\mathrm{T}+1$ condition, the mask (i.e., the next distractor) was presented directly after the target frame (Fig. 5a); in the $\mathrm{T}+$ 2 condition, a blank frame was inserted between the target and the mask (Fig. 5b).

Because the strength of masking is known to decrease as the target-mask SOA is increased, we expected the processing of the target to be harder in the $\mathrm{T}+1$ than in the $\mathrm{T}+2$ condition and the RT to be correspondingly longer.

Method

\section{Participants}

Fifteen undergraduate students participated for course credit. All reported normal or corrected-to-normal vision and were naïve as to the purpose of the experiment.

\section{Design and procedure}

The apparatus, design, and procedures were the same as those for lag 3 in Experiment 4, with the following exceptions. The RSVP sequence consisted of a leading stream of digits followed by a single letter target. There were two conditions. In the $\mathrm{T}+1$ condition, the target $(\mathrm{C}$ or $\mathrm{G})$ was followed directly by a mask (target-mask SOA $=100 \mathrm{~ms}$; Fig. 5a). In the $T+2$ condition, a blank frame was inserted
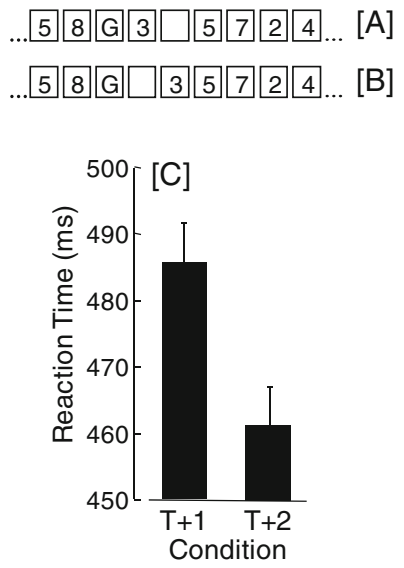

Fig. $5 \mathbf{a}$ and $\mathbf{b}$ Schematic representation of the sequence of events in the in the $T+1$ and the $T+2$ conditions, respectively. Each square represents one 7.14-ms frame. The small gap between each square represents a blank ISI of $93 \mathrm{~ms}$. c Mean RT, separately for each condition. Error bars indicate standard errors of the means between the target and the mask (target-mask SOA = $200 \mathrm{~ms}$; Fig. 5b). In both conditions, the mask was a digit chosen randomly from the set 2-9. A digit-mask was used, instead of the pattern mask used to mask T2 in the previous experiments, because the single target was intended to correspond to $\mathrm{T} 1$ in the previous experiments, in which the $\mathrm{T} 1$ mask was a digit. Beginning with the third posttarget frame, the display continued with more digit distractors until the participant responded or until the total number of frames in the RSVP stream reached 25 (Fig. 5a, b). Participants were instructed to identify the target letter as quickly and accurately as possible, by pressing the N-key when the target was $\mathrm{C}$ and the M-key when the target was G. There were 12 practice trials, followed by 100 trials for each of the two conditions, randomly intermixed.

Results and discussion

The RT scores, averaged across observers, are illustrated in Fig. $5 c$, separately for the $\mathrm{T}+1$ and $\mathrm{T}+2$ conditions. Only trials on which the target was identified correctly were included in the RT analysis. Mean accuracy in the $\mathrm{T}+1$ condition $(86.9 \%)$ did not differ significantly from that in the $\mathrm{T}+2$ condition $(88.9 \%), t(14)=1.37, p=.193$. A $t$-test for related samples confirmed that the mean RT was longer in the $\mathrm{T}+1$ condition, $t(14)=3.79, p=.002$. This result indicates that more time is required to identify a target when it is followed directly by a mask than when a blank frame intervenes between the target and the mask. This is consistent with the idea that a response ceiling concealed an actual difference in $\mathrm{T} 1$ processing difficulty - and hence, in T1-processing timebetween the $\mathrm{T} 1+1$ and the $\mathrm{T} 1+2$ conditions in Experiment 4 , with $\mathrm{T} 1$ processing taking longer in the $\mathrm{T} 1+1$ condition.

\section{References}

Akyürek, E. G., \& Hommel, B. (2005). Short-term memory and the attentional blink: Capacity versus content. Memory \& Cognition, $33,654-663$.

Akyürek, E. G., \& Hommel, B. (2006). Memory operations in rapid serial visual presentation. European Journal of Cognitive Psychology, 18, 520-536.

Akyürek, E. G., Hommel, B., \& Jolicœur, P. (2007). Direct evidence for a role of working memory in the attentional blink. Memory \& Cognition, 35, 621-627.

Anderson, J. R., \& Lebiere, C. (1998). The atomic components of thought. Mahwah: Erlbaum.

Bowman, H., \& Wyble, B. (2007). The simultaneous type, serial token model of temporal attention and working memory. Psychological Review, 114, 38-70.

Broadbent, D. E., \& Broadbent, M. H. (1987). From detection to identification: Response to multiple targets in rapid serial visual presentation. Perception \& Psychophysics, 42, 105113. 
Chun, M. M., \& Potter, M. C. (1995). A two-stage model for multiple target detection in serial visual presentation. Journal of Experimental Psychology. Human Perception and Performance, 21, 109-127.

Colzato, L. S., Spape, M. M. A., Pannebakker, M. M., \& Hommel, B. (2007). Working memory and the attentional blink: Blink size is predicted by individual differences in operation span. Psychonomic Bulletin \& Review, 14, 1051-1057.

Dell'Acqua, R., Jolicœur, P., Luria, R., \& Pluchino, P. (2009). Reevaluating encoding-capacity limitations as a cause of the attentional blink. Journal of Experimental Psychology. Human Perception and Performance, 35, 338-351.

Di Lollo, V., Kawahara, J., Ghorashi, S. M. S., \& Enns, J. T. (2005). The attentional blink: Resource depletion or temporary loss of control? Psychological Research, 69, 191-200.

Dux, P. E., Asplund, C. L., \& Marois, R. (2008). An attentional blink for sequentially presented targets: Evidence in favor of resource depletion accounts. Psychonomic Bulletin \& Review, 15(4), 809-813.

Dux, P. E., Asplund, C. L., \& Marois, R. (2009). Both exogenous and endogenous target salience manipulations support resource depletion accounts of the attentional blink: A reply to Olivers, Spalek, Kawahara, and Di Lollo (2009). Psychonomic Bulletin \& Review, 16(1), 219-224.

Dux, P. E., \& Coltheart, V. (2005). The meaning of the mask matters: Evidence of conceptual interference in the attentional blink. Psychological Science, 16, 775-779.

Dux, P. E., \& Marois, R. (2009). The attentional blink: A review of data and theory. Attention, Perception, \& Psychophysics, 71, 1683-1700.

Ghorashi, S., Enns, J. T., Spalek, T. M., \& Di Lollo, V. (2009). Spatial cuing does not affect the magnitude of the attentional blink. Attention, Perception, \& Psychophysics, 71, 989-993.

Ghorashi, S. M. S., Zuvic, S. M., Visser, T. A. W., \& Di Lollo, V. (2003). Focal distraction: Spatial shifts of attentional focus are not required for contingent capture. Journal of Experimental Psychology. Human Perception and Performance, 29, 78-91.

Giesbrecht, B., \& Di Lollo, V. (1998). Beyond the attentional blink: Visual masking by object substitution. Journal of Experimental Psychology. Human Perception and Performance, 24, 1454-1466.

Grandison, T. D., Ghirardelli, T. G., \& Egeth, H. E. (1997). Beyond similarity: Masking of the target is sufficient to cause the attentional blink. Perception \& Psychophysics, 59, 266-274.

Jannati, A., Spalek, T. M., \& Di Lollo, V. (2011). Neither backward masking of T2 nor task switching is necessary for the attentional blink. Psychonomic Bulletin \& Review, 18, 70-75.

Jannati, A., Spalek, T. M., Lagroix, H. E. P., \& Di Lollo, V. (2012). The attentional blink is not affected by backward masking of T2, T2mask SOA, or level of T2 impoverishment. Journal of Experimental Psychology. Human Perception and Performance, 38, 161-168.

Jolicœur, P. (1998). Modulation of the attentional blink by on-line response selection: Evidence from speeded and unspeeded Task decisions. Memory \& Cognition, 26, 1014-1032.

Jolicœur, P. (1999). Concurrent response-selection demands modulate the attentional blink. Journal of Experimental Psychology. Human Perception and Performance, 25, 1097-1113.

Jolicœur, P., \& Dell'Acqua, R. (1998). The demonstration of short-term consolidation. Cognitive Psychology, 36, 138-202.

Kanwisher, N. G. (1987). Repetition blindness: Type recognition without token individuation. Cognition, 27, 117-143

Kawahara, J., Kumada, T., \& Di Lollo, V. (2006). The attentional blink is governed by a temporary loss of control. Psychonomic Bulletin \& Review, 13, 886-890.

Lagroix, H. E. P., Spalek, T. M., \& Di Lollo, V. (2011). The role of observer strategy in the single-target AB paradigm. Psychonomic Bulletin \& Review, 18, 917-922.

Martens, S., \& Wyble, B. (2010). The attentional blink: Past, present, and future of a blind spot in perceptual awareness. Neuroscience and Biobehavioral Reviews, 34, 947-957.
McAuliffe, S. P., \& Knowlton, B. J. (2000). Dissociating the effects of featural and conceptual interference on multiple target processing in rapid serial visual presentation. Perception \& Psychophysics, 62, 187-195.

McLaughlin, E. N., Shore, D. I., \& Klein, R. M. (2001). The attentional blink is immune to masking-induced data limits. Quarterly Journal of Experimental Psychology. A, Human Experimental Psychology, 54A, 169-196.

Mozer, M. C. (1989). Types and tokens in visual letter perception. Journal of Experimental Psychology. Human Perception and Performance, 15(2), 287-303.

Nieuwenstein, M. R., \& Potter, M. C. (2006). Temporal limits of selection and memory encoding: A comparison of whole versus partial report in rapid serial visual presentation. Psychological Science, 17, 471-475.

Nieuwenstein, M. R., Potter, M. C., \& Theeuwes, J. (2009a). Unmasking the attentional blink. Journal of Experimental Psychology. Human Perception and Performance, 35, 159-169.

Nieuwenstein, M. R., Van der Burg, E., Theeuwes, J., Wyble, B., \& Potter, M. (2009b). Temporal constraints on conscious vision: On the ubiquitous nature of the attentional blink. Journal of Vision, 9 , $1-14$.

Olivers, C. N. L., Hulleman, J., Spalek, T. M., Kawahara, J.-I., \& Di Lollo, V. (2011). The sparing is far from spurious: Reevaluating within-trial contingency effects in the attentional blink. Journal of Experimental Psychology. Human Perception and Performance, 37(2), 396-408.

Olivers, C. N. L., \& Meeter, M. (2008). A boost and bounce theory of temporal attention. Psychological Research, 115, 836-863.

Olivers, C. N. L., Spalek, T. M., Kawahara, J.-I., \& Di Lollo, V. (2009). The attentional blink: Increasing target salience provides no evidence for resource depletion. A commentary on Dux, Asplund, and Marois (2008). Psychonomic Bulletin \& Review, 16(1), 214-218.

Olivers, C. N. L., van der Stigchel, S., \& Hulleman, J. (2007). Spreading the sparing: Against a limited-capacity account of the attentional blink. Psychological Research, 71, 126-139.

Olson, I. R., Chun, M. M., \& Anderson, A. K. (2001). Effects of phonological length on the attentional blink for words. Journal of Experimental Psychology. Human Perception and Performance, 27, 1116-1123.

Ouimet, C., \& Jolicœur, P. (2007). Beyond task 1 difficulty: The duration of T1 encoding modulates the attentional blink. Visual Cognition, 15, 290-304.

Phillips, W. A., \& Singer, W. (1974). Function and interaction of ON and OFF transients in vision: I. Psychophysics. Experimental Brain Research, 19, 493-506.

Potter, M. C., \& Levy, E. I. (1969). Recognition memory for a rapid sequence of pictures. Journal of Experimental Psychology, 81, 10 15 .

Potter, M. C., Nieuwenstein, M., \& Strohminger, N. (2008). Whole report versus partial report in RSVP sentences. Journal of Memory and Language, 58, 907-915.

Raymond, J. E., Shapiro, K. L., \& Arnell, K. M. (1992). Temporal suppression of visual processing in an RSVP task: An attentional blink? Journal of Experimental Psychology. Human Perception and Performance, 18, 849-860.

Seiffert, A. E., \& Di Lollo, V. (1997). Low-level masking in the attentional blink. Journal of Experimental Psychology. Human Perception and Performance, 23, 1061-1073.

Shapiro, K. L., Raymond, J. E., \& Arnell, K. M. (1994). Attention to visual pattern information produces the attentional blink in rapid serial visual presentation. Journal of Experimental Psychology. Human Perception and Performance, 20, 357-371.

Smallwood, J., \& Schooler, J. W. (2006). The restless mind. Psychological Bulletin, 132, 946-958. 
Spencer, T. J., \& Shuntich, R. (1970). Evidence for an interruption theory of backward masking. Journal of Experimental Psychology, 85, 198-203.

Taatgen, N. A., Juvina, I., Schipper, M., Borst, J. P., \& Martens, S. (2009). Too much control can hurt: A threaded cognition model of the attentional blink. Cognitive Psychology, 59, 1-29.

Taylor, M. M., \& Creelman, C. D. (1967). PEST: Efficient estimates on probability functions. The Journal of the Acoustical Society of America, 41, 782-787.

Visser, T. A. W. (2007). Masking T1 difficult: Processing time and the attentional blink. Journal of Experimental Psychology. Human Perception and Performance, 33, 285-297.

Visser, T. A. W., Bischof, W. F., \& Di Lollo, V. (2004). Rapid serial visual distraction: Task-irrelevant items can produce an attentional blink. Perception \& Psychophysics, 66, 1418-1432.
Wald, A. (1947). Sequential analysis. New York, NY: John Wiley \& Sons, Inc.

Ward, R., Duncan, J., \& Shapiro, K. (1996). The slow time-course of visual attention. Cognitive Psychology, 30, 79-109.

Ward, R., Duncan, J., \& Shapiro, K. (1997). Effects of similarity, difficulty, and nontarget presentation on the time course of visual attention. Perception \& Psychophysics, 59, 593-600.

Wyble, B., Bowman, H., \& Nieuwenstein, M. (2009). The attentional blink provides episodic distinctiveness: Sparing at a cost. Journal of Experimental Psychology. Human Perception and Performance, 35, 787-807.

Wyble, B., Potter, M. C., Bowman, H., \& Nieuwenstein, M. (2011). Attentional episodes in visual perception. Journal of Experimental Psychology. General, 140(3), 488-505. 